\title{
Low-Grade Inflammation and Increased Arterial Stiffness in Chinese Youth and Adolescents with Newly-Diagnosed Type 2 Diabetes Mellitus
}

\author{
Xin Li1, You-Ping Deng2, Miao Yang1, Yu-Wen Wu11, Su-Xin Sun1, Jia-Zhong Sun1 \\ ${ }^{1}$ Wuhan University Faculty of Medicine, Zhongnan Hospital, Clinic of Endocrinology, Wuhan, China \\ 2 Wuhan University Faculty of Medicine, Zhongnan Hospital, Clinic of Pediatrics, Wuhan, China
}

\section{WHAT IS ALREADY KNOWN ON THIS TOPIC?}

It is well known that adults with type 2 diabetes mellitus (T2DM) are at increased risk to develop cardiovascular disease due to the accelerated atherosclerosis. Adolescents with T2DM also appear to be at higher risk to develop atherosclerosis. Compared to their adolescent counterparts without diabetes, youth and adolescents with T2DM demonstrate increased arterial stiffness, a known precursor of atherosclerosis.

\section{WHAT THIS STUDY ADDS?}

We investigated whether low-grade inflammation correlates with arterial stiffness in Chinese youth and adolescents with newlydiagnosed T2DM.

\section{ABSTRACT}

Objective: To investigate the relationship between low-grade inflammation (LI) and increased arterial stiffness in Chinese youth and adolescents with newly-diagnosed type 2 diabetes mellitus (T2DM).

Methods: Ninety-eight subjects aged 10 to 24 years with newly-diagnosed T2DM were investigated for findings of general inflammation. Anthropometric measurements were taken. Data related to arterial stiffness [brachial artery distensibility (Branch D), augmentation index (Alx), carotid-femoral pulse wave velocity (CF-PWV)] were collected. The subjects were divided into a nonLI group (NLI, $n=42$ ) and a LI group ( $n=56$ ) according to their high-sensitivity C-reactive protein (Hs-CRP) levels.

Results: There were no significant differences in age and gender between the $\mathrm{LI}$ group and the NLI group. CF-PWV and Alx values of the LI group were higher than those of the $\mathrm{NLI}$ group $(p<0.01)$, while Branch $D$ values were lower in the $\mathrm{LI}$ group $(\mathrm{p}<0.01)$. Branch $\mathrm{D}, \mathrm{CF}-\mathrm{PWV}$, and Alx values correlated significantly with Hs-CRP overall $(r=-0.32,0.34,0.33$, all $p<0.01)$. Multivariate models revealed that in either group (LI or NLI), Hs-CRP, as a continuous variable, was an independent determinant of arterial stiffness parameters even after adjusting for other risk factors.

Conclusion: Newly-diagnosed T2DM youth and adolescents with LI present a more adverse cardiovascular disease risk profile and stiffer arteries. HsCRP levels correlated with arterial stiffness parameters and constituted an independent determinant of arterial stiffness.

Key words: Low-grade inflammation, youth and adolescent, type 2 diabetes mellitus, arterial stiffness

Conflict of interest: None declared

Received: 29.06 .2015

Accepted: 10.08 .2015

Address for Correspondence

Xin Li MD, Wuhan University Faculty of Medicine, Zhongnan Hospital, Clinic of Endocrinology, Wuhan, China Phone: 0086-27-67813107 E-mail:wdy2003win@163.com OJournal of Clinical Research in Pediatric Endocrinology, Published by Galenos Publishing. 


\section{Introduction}

A chronic low-grade inflammation (LI) and an activation of the immune system are observed in abdominal obesity and may have a role in the pathogenesis of obesity-related metabolic disorders, such as metabolic syndrome (MS), insulin resistance (IR), type 2 diabetes mellitus (T2DM), and cardiovascular disease (CVD) (1). It has been shown that systemic inflammatory markers are risk factors for the development of T2DM and its macrovascular complications (2).

With the economic development which took place in China over the past decades, the prevalence of childhood obesity has increased dramatically, ushering a variety of health problems including T2DM, which previously was not typically seen until much later in life (3). It is well known that adults with T2DM are at increased risk to develop CVD due to the accelerated atherosclerosis (4). Adolescents with T2DM also appear to be at higher risk to develop atherosclerosis. Compared to their adolescent counterparts without diabetes, youth and adolescents with T2DM demonstrate increased arterial stiffness, a known precursor of atherosclerosis (5).

To our knowledge, the correlations between LI and arterial stiffness have not been previously studied in Chinese youth and adolescents with newly-diagnosed T2DM. To address this issue, we evaluated arterial stiffness using pulse wave velocity (PWV), brachial artery distensibility (Brach D), and augmentation index (Alx) in a group of Chinese adolescents and young adults with newly-diagnosed T2DM and sought to determine whether high-sensitivity $\mathrm{C}$-reactive protein ( $\mathrm{Hs}-\mathrm{CRP}$ ), a marker of LI, correlated with arterial stiffness.

\section{Methods}

Ninety-eight participants (52 males and 46 females; mean age $18.4 \pm 4.2$ years) were recruited from among the inpatient and outpatient populations of the departments of Endocrinology and Pediatrics of the Zhongnan Hospital at Wuhan University. Eligibility criteria included presence of a newly-diagnosed T2DM state in subjects aged from 10 to 24 years who were free of the acute complications of diabetes such as diabetic ketoacidosis (DKA), and who also had no history or evidence of infectious diseases, autoimmune diseases, use of glucocorticoids in the past 3 months, and other relevant medical histories. Pregnant females were also excluded from this study. The patients were divided into LI group and non-LI group (NLI), according to their $\mathrm{Hs}-\mathrm{CRP}$ levels. A cut-off value of $2 \mathrm{mg} / \mathrm{L}$ for the $\mathrm{Hs}-\mathrm{CRP}$ values was accepted to define subjects with LI (6).

The diagnosis of T2DM was based on the American Diabetes Association criteria (7) and the guidelines recommended by the American Academy of Pediatrics (8). Diabetes was defined as: 1) Hemoglobin A1c (HbA1c) $\geq 6.5 \%$ (test performed in an appropriately certified laboratory); or 2) (defined as no caloric intake for at least 8 hours) fasting plasma glucose (FPG) $\geq 7.0 \mathrm{mmol} / \mathrm{L}$; or 3) 2-hour plasma glucose $\geq 11.1 \mathrm{mmol} / \mathrm{L}$ during an oral glucose tolerance test (OGTT) performed as described by the World Health Organization by using a glucose load containing the equivalent of $75 \mathrm{~g}$ anhydrous glucose dissolved in water; or 4) a random plasma glucose level of $11.1 \mathrm{mmol} / \mathrm{L}$ with symptoms of hyperglycemia. In the absence of unequivocal hyperglycemia, criteria 1-3 were confirmed by repeat testing. None of the patients had evidence of another specific type of diabetes. All subjects were islet cell antibodynegative (glutamic acid decarboxylase, islet cell antigen, insulin auto-antibodies).

All study procedures were approved by the Human Research Ethics Committee of Zhongnan Hospital, Wuhan University. Prior to enrollment in the study, written informed consent was obtained from subjects $\geq 18$ years old and from the parents or guardians for subjects $<18$ years old.

All the participants were given a questionnaire which included questions on the name, age, gender, occupation, smoking and drinking history, medical history, and family history of the individual. The average of two consecutive measurements of height and weight were used in the analyses. Body mass index (BMI) was calculated as weight $(\mathrm{kg}) / \mathrm{height}$ $(\mathrm{m})^{2}$. The average of 3 measurements of systolic blood pressure (SBP) and diastolic blood pressure (DBP), performed by a registered nurse using a mercurial sphygmomanometer with the subjects in a resting state, was used.

After a minimum 10-h fast, blood samples were collected. FPG was measured by using an Olympus AU5400 automatic biochemical analyzer with intra-assay and inter-assay coefficients of variation of $1.2 \%$ and $1.6 \%$. Fasting plasma insulin (FINS) was analyzed by using a Siemens immune 1000 automatic chemiluminescence analyzer. Homeostasis model assessment of IR (HOMA-IR) was calculated as FINSxFPG/22.5. Hs-CRP was measured by using a high-sensitivity enzyme-linked immunosorbent assay. Lipid profiles were performed by using an Olympus AU5400 automatic biochemical analyzer.

\section{Arterial Stiffness Measurements}

Brach D, carotid-femoral PWV (CF-PWV), and Alx were used as measures of arterial stiffness (9). Data of Brach D were obtained by a DynaPulse Pathway Instrument (PulseMetric, San Diego, CA, USA), which derived brachial artery pressure curves from arterial pressure signals obtained from a standard cuff sphygmomanometer assuming a straight tube brachial artery and T-tube aortic system. The coefficients of variability in this study were $<9 \%$.

A SphygmoCor SCOR-PVx System (Atcor Medical, Sydney, Australia) was used for heart rate (HR), CF-PWV, and Alx. The distance from carotid to femoral artery was measured to the nearest $0.1 \mathrm{~cm}$ twice, was averaged, and entered into the software. A tonometer was used to collect carotid and femoral artery waveforms gated by the R-wave on a simultaneously recorded electrocardiogram. CF-PWV was then calculated 
as the distance measured above divided by the time delay measured between the feet of the two waveforms reported in $\mathrm{m} / \mathrm{sec}$. CF-PWV of each subject was measured three times and averaged. The coefficients of variability in this study were $<8 \%$.

Alx is derived from the central pressure waveform by calculating the difference between the main outgoing wave and the reflected wave of the central arterial waveform, expressed as a percentage of the central pulse pressure. Alx was adjusted to a standard HR of 75 beats per minute to eliminate the influence of HR. The intra-class correlation coefficients were 0.7 to 0.9 .

\section{Statistical Analysis}

The analyses were performed with SPSS 19.0. The data were expressed as means \pm standard deviation (SD) for continuous variables, and as ratios for categorical variables. A t-test for continuous variables and $\mathrm{X}^{2}$ analyses for categorical variables were performed to determine whether differences in mean levels of variables existed between the two groups. Bivariate correlations were calculated between arterial stiffness variables and other covariates overall. General Linear Models were constructed using important covariates from correlation analyses to elucidate if $\mathrm{LI}$ was an independent determinate of arterial stiffness. A p value $<0.05$ was deemed significant statistically.

\section{Results}

Cardiovascular Disease Risk Factors and Arterial Stiffness Parameters by Groups

There were no significant differences in age and gender between the $\mathrm{LI}$ and $\mathrm{NLI}$ groups. Compared to the NLI group, CVD risk factors such as BMI, SBP, DBP, HOMA$I R$, triglyceride (TG), total cholesterol (TC), and low-density lipoprotein cholesterol (LDL-C) were all increased significantly in the $\mathrm{LI}$ group $(p<0.05$ or $p<0.01)$. The high-density lipoprotein cholesterol (HDL-C) values were alse significantly lower in the of $\mathrm{LI}$ group $(\mathrm{p}<0.01)$. The differences in FPG and $\mathrm{HbA} 1 \mathrm{c}$ between the two groups were not significant. CF-PWW and Alx of $\mathrm{LI}$ group were higher than those of the NLI group $(p<0.01)$, while Branch D of the LI group was lower than that of the NLI group $(p<0.01)$, indicating that the arterial stiffness of $L I$ group was higher than that of the NLI group (Table 1).

\section{Correlations Between High-Sensitivity C-Reactive Protein and} Arterial Stiffness Parameters

The three arterial stiffness parameters (Branch D, CF-PWV, and Alx) correlated with each other strongly (for all, $\mathrm{p}<0.01$ ). Branch D, CF-PWV, and Alx also correlated significantly with Hs-CRP overall $(r=-0.32,0.34$, and 0.33 ; for all, $p<0.01)$. Arterial stiffness parameters also significantly correlated with BMI, SBP, DBP, TG, TC, LDL-C, HDL-C, HbA1c, and HOMA-IR (for all, $p<0.05$ or $p<0.01)$, after the control of which, the coefficient correlation of Hs-CRP with Branch D, CF-PWV, and Alx was found to be $-0.11,0.13$, and 0.13 , respectively (for all, $p<0.05$ ).

\section{Multivariable Models for Independent Determinants of Arterial Stiffness}

Multivariate models revealed that in either group ( $\mathrm{LI}$ or NLI), Hs-CRP as a continuous variable was an independent determinant of arterial stiffness, demonstrated as Branch $D$, CF-PWV, and Alx, even after adjusting for BMI, SBP, DBP, TG, TC, LDL-C, HDL-C, FPG, HbA1c, and HOMA-IR. The other independent predictors of the arterial stiffness parameters are shown in Table 2.

\section{Discussion}

This study demonstrated that a more adverse CVD risk profile and stiffer arteries are found in newly-diagnosed T2DM youth and adolescents with LI. Hs-CRP, the marker of LI, may

\begin{tabular}{|c|c|c|c|c|c|c|c|c|}
\hline Group & $N$ & $\begin{array}{l}\text { Age } \\
\text { (years) }\end{array}$ & $\begin{array}{l}\text { Gender } \\
\text { (Male/Female) }\end{array}$ & $\begin{array}{l}\text { BMI } \\
\left(\mathrm{kg} / \mathrm{m}^{2}\right)\end{array}$ & $\begin{array}{l}\text { SBP } \\
(\mathrm{mmHg})\end{array}$ & $\begin{array}{l}\text { DBP } \\
(\mathrm{mmHg})\end{array}$ & $\begin{array}{l}\mathrm{TG} \\
(\mathrm{mmol} / \mathrm{L})\end{array}$ & $\begin{array}{l}\text { TC } \\
(\mathrm{mmol} / \mathrm{L})\end{array}$ \\
\hline NLI & 42 & $17.9 \pm 3.4$ & $22 / 20$ & $23.5 \pm 2.9$ & $112.6 \pm 11.2$ & $73.1 \pm 6.6$ & $1.23 \pm 0.22$ & $4.12 \pm 0.51$ \\
\hline LI & 56 & $18.8 \pm 4.7$ & $30 / 26$ & $25.7 \pm 3.2$ & $121.8 \pm 13.6$ & $79.5 \pm 7.5$ & $1.46 \pm 0.30$ & $4.62 \pm 0.65$ \\
\hline $\operatorname{tor} X^{2}$ & ND & 0.934 & 0.14 & 3.504 & 3.568 & 4.397 & 4.192 & 4.122 \\
\hline$p$ & ND & 0.352 & 0.907 & 0.001 & 0.001 & 0.000 & 0.000 & 0.000 \\
\hline Group & $\begin{array}{l}\text { LDL-C } \\
\text { (mmol/L) }\end{array}$ & $\begin{array}{l}\text { HDL-C } \\
(\mathrm{mmHg})\end{array}$ & $\begin{array}{l}\text { FPG } \\
(\mathrm{mmol} / \mathrm{L})\end{array}$ & $\begin{array}{l}\text { HbA1c } \\
(\%)\end{array}$ & HOMA-IR & $\begin{array}{l}\text { Branch D } \\
(\% / \mathrm{mmHg})\end{array}$ & $\begin{array}{l}\text { CF-PWV } \\
(\mathrm{m} / \mathrm{s})\end{array}$ & $\begin{array}{l}\text { Alx } \\
\text { (\%) }\end{array}$ \\
\hline NLI & $2.34 \pm 0.43$ & $1.42 \pm 0.31$ & $8.03 \pm 0.80$ & $7.75 \pm 2.0$ & $2.86 \pm 0.29$ & $7.02 \pm 1.36$ & $6.1 \pm 1.3$ & $-0.48 \pm 10.05$ \\
\hline LI & $3.01 \pm 0.62$ & $1.09 \pm 0.18$ & $8.19 \pm 0.81$ & $8.3 \pm 2.1$ & $4.15 \pm 0.32$ & $5.98 \pm 1.25$ & $7.6 \pm 1.8$ & $3.52 \pm 9.53$ \\
\hline $\mathrm{t}$ & 6.001 & 6.622 & 0.973 & 1.309 & 20.549 & 3.925 & 4.577 & 2.009 \\
\hline$p$ & 0.000 & 0.000 & 0.333 & 0.194 & 0.000 & 0.002 & 0.000 & 0.047 \\
\hline \multicolumn{9}{|c|}{$\begin{array}{l}\text { NLI: non-low-grade inflammation group, LI: low-grade inflammation group, BMI: body mass index, SBP: systolic blood pressure, DBP: diastolic blood pressure, TG: triglyceride, } \\
\text { TC: total cholesterol, LDL-C: low-density lipoprotein cholesterol, HDL-C: high-density lipoprotein cholesterol, FPG: fasting plasma glucose, HbA1c: hemoglobin A1c, HOMA-IR: } \\
\text { homeostasis model assessment of insulin resistance, Branch D: brachial artery distensibility, CF-PWV: carotid-femoral pulse wave velocity, Alx: augmentation index }\end{array}$} \\
\hline
\end{tabular}


Table 2. Multivariable models for independent determinants of arterial stiffness

\begin{tabular}{|c|c|c|c|}
\hline Variable & $\begin{array}{l}\text { Branch D } \\
\text { (Lower=Stiffer) }\end{array}$ & $\begin{array}{l}\text { CF-PWV } \\
\text { (Higher=Stiffer) }\end{array}$ & $\begin{array}{l}\text { Alx } \\
\text { (Higher=Stiffer) }\end{array}$ \\
\hline Intercept & 3.12 & 0.18 & -12.46 \\
\hline BMI & -0.18 & 0.11 & 0.15 \\
\hline SBP & & 0.0046 & 0.0075 \\
\hline DBP & -0.0075 & & \\
\hline LDL-C & & 0.0057 & 0.0086 \\
\hline HDL-C & 0.0087 & & -0.0025 \\
\hline Hs-CRP & -0.36 & 0.29 & 0.34 \\
\hline HOMA-IR & -0.0036 & & \\
\hline $\mathrm{R}^{2}$ & 0.38 & 0.47 & 0.29 \\
\hline \multicolumn{4}{|c|}{$\begin{array}{l}\text { BMI: body mass index, SBP: systolic blood pressure, DBP: diastolic blood pressure, } \\
\text { LDL-C: low-density lipoprotein cholesterol, HDL-C: high-density lipoprotein cholesterol, } \\
\text { HOMA-IR: homeostasis model assessment of insulin resistance, Branch D: brachial } \\
\text { artery distensibility, CF-PWV: carotid-femoral pulse wave velocity, Alx: augmentation } \\
\text { index } \\
\text { Values are } \beta \text { coefficients for significant covariates left in the model after stepwise } \\
\text { regression. All } p<0.01\end{array}$} \\
\hline
\end{tabular}

be especially helpful in predicting increased arterial stiffness in young individuals with newly-diagnosed T2DM. These data also suggest that newly-diagnosed T2DM youth and adolescents require aggressive interventions to prevent atherosclerosisrelated diseases. To our knowledge, this is the first report about the relationship between $\mathrm{LI}$ and arterial stiffness in young and adolescent T2DM patients.

In adults, the correlation between inflammation and T2DM has been well demonstrated. Subclinical chronic inflammation seems to be an independent risk factor for the development of T2DM. It has been reported that high levels of many inflammatory factors at baseline in diverse human populations are correlated with the incidence of T2DM (10). Prospective studies have identified white blood cell count (11), proinflammatory cytokines (12), chemokines (13), and other several indirect markers of inflammation, such as fibrinogen, sialic acid, and plasminogen activator inhibitor-1 (14), as predictors of T2DM. Amongst all inflammatory biomarkers, Hs-CRP measurement stands out as the least expensive, better standardized, and widely available (15). We have therefore, in this study, used Hs-CRP to reflect the status of inflammation in young individuals with newly-diagnosed T2DM and found that the cut-off point of $\mathrm{LI}$ was $2 \mathrm{mg} / \mathrm{L}$, a finding in accordance with other reports (6).

It is well known that increased arterial stiffness is an independent predictor of CVD and that it is not related to blood pressure (16). The importance of inflammation on the pathogenesis of arterial stiffness has been widely studied in adults $(17,18)$. Arterial stiffness is associated with increased activity of angiotensin II (Ang II), which results in increased
NADPH oxidase activity, reduced NO bioavailability, and increased production of reactive oxygen species (19). Ang II not only activates matrix metalloproteinases which degrade transforming growth factor $\beta$ (TGF- $\beta$ ) precursors to produce active TGF- $\beta$, resulting in increased arterial fibrosis, but also activates cytokines, including monocyte chemoattractant protein-1, tumor necrosis factor- $\alpha$, interleukin-1 (IL-1), IL-17 and IL-6 (20). There are also some clinical evidence that indicate the association of inflammation with increased arterial stiffness. In a 20-year follow-up from the Caerphilly prospective study, conducted on a predominantly Caucasian cohort of 825 men who underwent baseline and follow-up PWV measurements, it has been demonstrated that the only independent predictors of the PWV at follow-up were pulse pressure, CRP, glucose levels, and waist circumference. Among the clinical variables, cumulative exposure to CRP was the variable with the strongest association (21). We also found in this study that Hs-CRP significantly correlated with arterial stiffness parameters and was an independent determinant of arterial stiffness.

Patients with diabetes carry a two to fourfold increased risk of developing CVD. Patients with T2DM also have other CVD risk factors, such as hypertension, dyslipidemia, obesity, and endothelial dysfunction (22). Not only is the incidence of CVD higher in diabetes, the mortality of the diabetic patients after a cardiac event is also significantly increased in comparison with non-diabetics (23). In the past decades, the incidence of T2DM in children and adolescents has shown a progressive increase (8). It has been demonstrated that children and adolescents with T2DM are at greater risk for the development of atherosclerotic CVD than the general population. Naylor et al (24) reported that carotid intima-medial thickness in adolescents with T2DM was increased as compared to both obese and lean control subjects, while flow-mediated dilation in the T2DM group was significantly decreased compared to that of lean group, suggesting impairment of vascular health in adolescents with T2DM. Urbina et al (25) found that arterial stiffness of adolescents and young adults with obesity-related T2DM was increased and also reported that these abnormalities persisted after controlling for factors such as blood pressure and lipids.

Although some research on the relationship of T2DM and arterial stiffness in adolescents has been conducted, the factors influential in this relationship are largely unknown. In a study on healthy adolescents and young adults, it was reported that although IR is associated with increased arterial stiffness, traditional cardiovascular risk factors, especially obesity and blood pressure, were the major determinants of arterial stiffness in such individuals (26). These authors also found that TG/HDL-C, an estimate of small-dense LDL-C, was an independent determinant of arterial stiffness in adolescents and young adults, especially in obese subjects (27). In young individuals and adolescents with newly-diagnosed T2DM, we are the first to report that $\mathrm{LI}$ was an independent determinant of arterial stiffness. 
It has been well established that obesity is an independent determinant of CVD. Arterial stiffness has been reported in individuals with obesity or $\mathrm{MS}$, and weight reduction may decrease arterial stiffness (28). In a study aiming to investigate the influence of adiposis on the progression of arteriosclerosis in the early teens, it was found that the changes in BMI values correlated positively with systolic and DBP and with PWV, indicating the tight relationship between obesity and arterial stiffness even in young individuals and in adolescents (29). It should be mentioned that BMI and Hs-CRP were both independent predictors of the arterial stiffness parameters in multivariate models in this present study (as shown in Table 2). We also found that the BMI correlated significantly with Hs-CRP levels (data not shown). However, after controlling for $\mathrm{BMI}$ and other risk factors, Hs-CRP still correlated with Branch D, CF-PWV, and Alx significantly, and in multivariate models, Hs-CRP was an independent determinant of Branch D, CF-PWV, and Alx even after adjusting for BMI and other factors. These results indicated that Hs-CRP correlated with arterial stiffness parameters and was an independent determinant of arterial stiffness. To measure Hs-CRP may be a useful tool to screen for those at higher risk for CVD among the newlydiagnosed T2DM youth and adolescents.

We realize that this study has some limitations. The smallness of the sample size is one of these limitations. Our cross-sectional design does not allow us to determine whether $\mathrm{Hs}-\mathrm{CRP}$ is a causal factor of arterial stiffness or just a satellite phenomenon. In addition, we did not assess other "nontraditional" risk factors which may also contribute to increased vascular stiffness such as small LDL particles, lipoprotein (a), serum homocysteine, and endothelin-1.

On the whole, the findings of this study have revealed that newly-diagnosed T2DM youth and adolescents with $\mathrm{LI}$ had a more adverse CVD risk profile and stiffer arteries. Hs-CRP correlated with all three arterial stiffness parameters, namely, Branch D, CF-PWV, and Alx, and was an independent determinant of arterial stiffness after adjustment for BMI, SBP, DBP, TG, TC, LDL-C, HDL-C, FPG, HbA1c, and HOMA-IR. The findings also showed that Hs-CRP, the marker of $\mathrm{LI}$, may be helpful in predicting increased arterial stiffness in newlydiagnosed T2DM youth and adolescents.

\section{Acknowledgements}

This research was supported by the Hubei Province health and family planning scientific research project (WJ20150018). We would like to acknowledge all the members of the Department of Endocrinology and Pediatrics of Zhongnan Hospital Wuhan University. We would also like to thank the participants of this study and their families.

\section{Authorship Contributions}

Ethics Committee Approval: Zhongnan Hospital, Wuhan University Ethics Committee (Approval number: 2012-086),
Informed Consent: It was taken, Concept: Xin Li, Design: Xin Li, Data Collection or Processing: Xin Li, You-Ping Deng, Miao Yang, Yu-Wen Wu, Analysis or Interpretation: Xin Li, Su-Xin Sun, Jia-Zhong Sun, Literature Search: Xin Li, You-Ping Deng, Writing: Xin Li, Peer-review: Externally peer-reviewed, Financial Disclosure: This research was supported by the Hubei Province health and family planning scientific research project (WJ20150018).

\section{References}

1. Lee BC, Lee J. Cellular and molecular players in adipose tissue inflammation in the development of obesity-induced insulin resistance. Biochem Biophys Acta 2014;1842:446462. Epub 2013 May 22

2. Cruz NG, Sousa LP, Sousa MO, Pietrani NT, Fernandes $A P$, Gomes KB. The linkage between inflammation and Type 2 diabetes mellitus. Diabetes Res Clin Pract 2013;99:85-92. Epub 2012 Dec 14

3. Yang W, Lu J, Weng J, Jia W, Ji L, Xiao J, Shan Z, Liu J, Tian $\mathrm{H}$, Ji Q, Zhu D, Ge J, Lin L, Chen L, Guo X, Zhao Z, Li Q, Zhou Z, Shan G, He J; China National Diabetes and Metabolic Disorders Study Group. Prevalence of diabetes among men and women in China. N Engl J Med 2010;362:1090-1101.

4. Rock CL, Flatt SW, Pakiz B, Taylor KS, Leone AF, Brelje K, Heath DD, Quintana EL, Sherwood NE. Weight loss, glycemic control, and cardiovascular disease risk factors in response to differential diet composition in a weight loss program in type 2 diabetes: a randomized controlled trial. Diabetes Care 2014;37:1573-1580. Epub 2014 Apr 23

5. Christen Al, Armentano RL, Miranda A, Graf S, Santana DB, Zocalo Y, Baglivo HP, Sanchez RA. Arterial wall structure and dynamics in type 2 diabetes mellitus methodological aspects and pathophysiological findings. Curr Diabetes Rev 2010;6:367-377.

6. Soisson V, Brailly-Tabard S, Empana JP, Feart C, Ryan J, Bertrand M, Guiochon-Mantel A, Scarabin PY. Low plasma testosterone and elevated carotid intima-media thickness: importance of low-grade inflammation in elderly men. Atherosclerosis 2012;223:244-249. Epub 2012 May 16

7. American Diabetes Association. Standards of medical care in diabetes-2013. Diabetes Care 2013;36(Suppl 1):11-66.

8. Copeland KC, Silverstein J, Moore KR, Prazar GE, Raymer T, Shiffman RN, Springer SC, Thaker VV, Anderson M, Spann SJ, Flinn SK; American Academy of Pediatrics. Management of newly diagnosed type 2 Diabetes Mellitus (T2DM) in children and adolescents. Pediatrics 2013;131:364-382. Epub 2013 Jan 28

9. Shah AS, Dolan LM, Gao Z, Kimball TR, Urbina EM. Racial differences in arterial stiffness among adolescents and young adults with type 2 diabetes. Pediatr Diabetes 2012;13:170-175. Epub $2011 \mathrm{Jul} 25$

10. Donath MY. Targeting inflammation in the treatment of type 2 diabetes. Diabetes Obes Metab 2013;15(Suppl 3):193-196.

11. Duncan BB, Schmidt MI, Pankow JS, Ballantyne CM, Couper D, Vigo A, Hoogeveen R, Folsom AR, Heiss G; Atherosclerosis Risk in Communities Study. Low-grade systemic inflammation and the development of type 2 diabetes: the atherosclerosis risk in communities study. Diabetes 2003;52:1799-1805.

12. Spranger J, Kroke A, Möhlig M, Hoffmann K, Bergmann MM, Ristow M, Boeing $\mathrm{H}$, Pfeiffer AF. Inflammatory cytokines and 
the risk to develop type 2 diabetes: results of the prospective population-based European Prospective Investigation into Cancer and Nutrition (EPIC)-Potsdam Study. Diabetes 2003;52:812-817.

13. Herder C, Baumert J, Thorand B, Koenig W, de Jager $W$, Meisinger $\mathrm{C}$, Illig $\mathrm{T}$, Martin $\mathrm{S}$, Kolb $\mathrm{H}$. Chemokines as risk factors for type 2 diabetes: results from the MONICA/KORA Augsburg study, 1984-2002. Diabetologia 2006;49:921-929. Epub 2006 Mar 11

14. Festa A, Williams K, Tracy RP, Wagenknecht LE, Haffner SM. Progression of plasminogen activator inhibitor-1 and fibrinogen levels in relation to incident type 2 diabetes. Circulation 2006;113:1753-1759. Epub 2006 Apr 3

15. Wang $X$, Bao W, Liu J, Ouyang YY, Wang D, Rong S, Xiao $X$, Shan ZL, Zhang $Y$, Yao P, Liu LG. Inflammatory markers and risk of type 2 diabetes: a systematic review and metaanalysis. Diabetes Care 2013;36:166-175.

16. Dabelea $D$, Talton JW, D'Agostino R Jr, Wadwa RP, Urbina EM, Dolan LM, Daniels SR, Marcovina SM, Hamman RF. Cardiovascular risk factors are associated with increased arterial stiffness in youth with type 1 diabetes: the SEARCH CVD study. Diabetes Care 2013;36:3938-3943. Epub 2013 Oct 7

17. Park S, Lakatta EG. Role of inflammation in the pathogenesis of arterial stiffness. Yonsei Med J 2012;53:258-261.

18. Wang M, Zhang J, Jiang LQ, Spinetti G, Pintus G, Monticone $R$, Kolodgie FD, Virmani R, Lakatta EG. Proinflammatory profile within the grossly normal aged human aortic wall. Hypertension 2007;50:219-227. Epub 2007 Apr 23

19. Kajiya M, Hirota $M$, Inai $Y$, Kiyooka T, Morimoto T, Iwasaki T, Endo K, Mohri S, Shimizu J, Yada T, Ogasawara Y, Naruse $\mathrm{K}$, Ohe T, Kajiya F. Impaired NO-mediated vasodilation with increased superoxide but robust EDHF function in right ventricular arterial microvessels of pulmonary hypertensive rats. Am J Physiol Heart Circ Physiol 2007;292:2737-2744. Epub 2007 Jan 12

20. Csiszar A, Ungvari Z, Koller A, Edwards JG, Kaley G. Proinflammatory phenotype of coronary arteries promotes endothelial apoptosis in aging. Physiol Genomics 2004;17:2130.

21. McEniery CM, Spratt M, Munnery M, Yarnell J, Lowe GD, Rumley A, Gallacher J, Ben-Shlomo Y, Cockcroft JR, Wilkinson IB. An analysis of prospective risk factors for aortic stiffness in men: 20-year follow-up from the Caerphilly prospective study. Hypertension 2010;56:36-43. Epub 2010 Jun 7

22. Norhammar A, Schenck-Gustafsson K. Type 2 diabetes and cardiovascular disease in women. Diabetologia 2013;56:1-9. Epub 2012 Sep 4

23. Fox CS. Cardiovascular disease risk factors, type 2 diabetes mellitus, and the Framingham Heart Study. Trends Cardiovasc Med 2010;20:90-95.

24. Naylor LH, Green DJ, Jones TW, Kalic RJ, Suriano KL, Shah M, Hopkins N, Davis EA. Endothelial function and carotid intima-medial thickness in adolescents with type 2 diabetes mellitus. J Pediatr 2011;159:971-974. Epub 2011 Jun 30

25. Urbina EM, Kimball TR, Khoury PR, Daniels SR, Dolan LM. Increased arterial stiffness is found in adolescents with obesity or obesity-related type 2 diabetes mellitus. J Hypertens 2010;28:1692-1698.

26. Urbina EM, Gao Z, Khoury PR, Martin LJ, Dolan LM. Insulin resistance and arterial stiffness in healthy adolescents and young adults. Diabetologia 2012;55:625-631. Epub 2011 Dec 23

27. Urbina EM, Khoury PR, McCoy CE, Dolan LM, Daniels $\mathrm{SR}$, Kimball TR. Triglyceride to HDL-C ratio and increased arterial stiffness in children, adolescents, and young adults. Pediatrics 2013;131:1082-1090. Epub 2013 Mar 4

28. Leopold JA. Cellular and molecular mechanisms of arterial stiffness associated with obesity. Hypertension 2013;62:1003-1004. Epub 2013 Sep 23

29. Kudo U, Takahashi I, Matsuzaka M, Umeda T, Kitagawa $N$, Kudo H, Chiba Y, Sasaki E, Nishimura M, Nakaji S. Influence of obesity on blood pressure and arterial stiffness in the early teens. Obes Res Clin Pract 2013;7:211-217. 